\title{
Linear Time-of-Flight Mass Spectrometers: Postsource Pulse Focusing Conditions and Mass Scale
}

\author{
Damaschin Ioanoviciu \\ Institute for Isotopic and Molecular Technology, Cluj-Napoca, Romania
}

The condition to cancel aberrations that result from initial ion velocities and length of ionization process by postsource pulse focusing in linear time-of-flight mass spectrometers is derived. The ion arrival time distribution as a function of ion mass is given, which permits mass scale calibration. Simple formulas allow calculation of the voltage needed for postsource pulse focusing and the limits of the mass range where the focusing action is felt. The theory is illustrated for a specific instrument case. (f Am Soc Mass Spectrom 1995, 6, 889-891)

$\mathrm{T}$ To improve the resolution of linear time-of-flight mass spectrometers (TOFMS) variable electric fields have been applied to ion drift regions between the ion source and detector. Velocity compaction [1], postsource pulse focusing (PSPF) [2], and dynamic field focusing [3] are all such procedures that have been presented in literature. PSPF has been modeled by computer [2], experimentally demonstrated for gas-phase ionization [4], and mass spectra with resolutions of 2900 have been recorded for laser desorbed ions produced in the TOFMS instrument described in ref 5 .

The aim of the present short communication is to derive the focusing condition and mass scale as well as to give simple formulas to calculate the instrumental parameters needed for implementation of PSPF.

The flight times over various ion acceleration regions were calculated by using the formulas of ref 6 , and are rewritten in terms of velocities:

$$
\begin{aligned}
& t=(m /(z e F))\left(v_{\text {out }}-v_{\text {in }}\right) \\
& \tau_{\text {uut }}=\left(v_{\text {ill }}^{2}+2 z e F L / / m\right)^{1 / 2}
\end{aligned}
$$

where $t$ is the time spent by the ion traveling across a region of length $L$ while accelerated by an electric field of strength $F$. The ion of mass $m$ and $z$ elementary charges $e$ enters and leaves the region with longitudinal velocity $v_{\text {in }}$ and $v_{\text {out }}$, respectively. Let us consider an ion created at a distance $s_{0}$ from the ion source reference plane $P$ (Figure 1 ) that has initial longitudinal velocity $v_{0}$ directed toward the detector at a moment $t_{0}$ after the beginning of the ionization event, taken as the time origin. The source reference plane is

Address reprint requests to Dr. D. Loanoviciu. Institute of Isotopic and Molecular Techrolingy, P.O. Box 700. [R-3400 Cluj-Napoca Romania. normal to the ion packet movement direction, in the middle of the ionization region. The reference ion of mass $m$, created at the time origin and starting from rest, from the source reference plane will be detected $t_{\text {or }}$ later. By using (1) and (2) twice we find the first order approximation for the time $t_{2}$ needed by this ion to travel through the source and secondary acceleration regions, where constant fields were applied:

$$
t_{2}=\left(D_{\mathrm{s}} / v_{\mathrm{s}}\right)\left[1+s_{0} k /\left(2 D_{1} \sigma^{2}\right)\right]-m v_{0} /\left(z e F_{1}\right)
$$

while the ion longitudinal velocity is there:

$$
v_{2}=v_{\mathrm{s}}\left[1+s_{0} /\left(2 \sigma^{2} D_{1}\right)\right]
$$

The symbols are defined in Table 1 and by the relations

$$
\begin{aligned}
D_{s} & =2 \sigma\left[D_{1}+D_{2} /(1+\sigma)\right] \\
\sigma^{2} & =U_{\mathrm{s}} /\left(e F_{1} D_{1}\right) \\
v_{\mathrm{s}} & =\left(2 U_{\mathrm{s}} / m\right)^{1 / 2} \\
k & =\sigma^{2}\left[D_{1}(1+\sigma)-D_{2} / \sigma\right] /\left[D_{1}(1+\sigma)+D_{2}\right]
\end{aligned}
$$

The PSPF field is established between the planes $F$ and $G$, at a moment $T$ from the time origin. Because the ion was allowed to drift freely along $v_{2}\left(T-t_{0}-\right.$ $t_{2}$ ), it needs time $t_{3}$ to travel the remaining $D_{3}$ after the PSPF field is applied:

$$
t_{3}=m\left(v_{3}-v_{2}\right) /\left(z e F_{3}\right)
$$

The ion velocity at the plane $G$ is increased to $v_{3}$ :

$$
v_{3}=\left\{v_{2}^{2}+\left(2 z e F_{3} / m\right)\left[D_{3}-v_{2}\left(T-t_{0}-t_{2}\right)\right]\right\}^{1 / 2}
$$




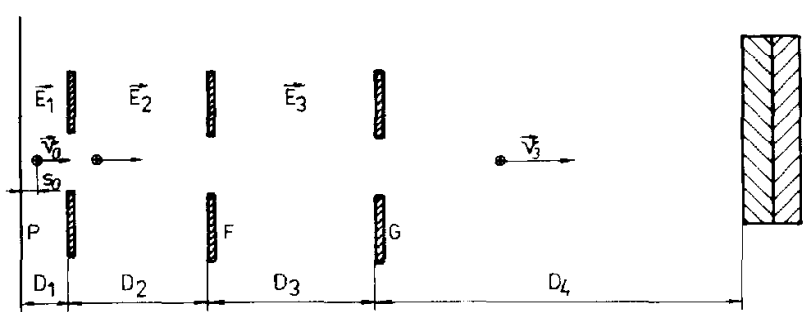

Figure 1. Linear time-of-flight mass spectrometer with postsource focusing grid: basic geometric parameters.

Table 1. Definitions of symbols used in text

\begin{tabular}{ll}
\hline Symbol & Physical meaning $^{\text {a }}$ \\
\hline \hline$D_{1}$ & Source region length \\
$D_{2}$ & Secondary acceleration region length \\
$D_{3}$ & PSPF region length \\
$D_{4}$ & PSPF grid-detector distance \\
$s_{0}$ & Initial ion distance to the reference plane \\
$F_{1}$ & Source region field strength \\
$F_{2}$ & Secondary acceleration region field strength \\
$F_{3}$ & PSPF region field strength \\
$v_{\mathrm{s}}$ & Reference ion velocity at source exit \\
$v_{1}$ & Reference ion velocity before detection \\
$v_{0}$ & Initial ion velocity \\
$U_{\mathrm{s}}$ & Reference ion energy at source exit \\
$U_{f}$ & Reference ion energy before detection \\
$t_{\mathrm{f} 0}$ & Reference ion total flight time \\
$t_{0}$ & Instant when ion created \\
\hline
\end{tabular}

${ }^{a}$ The reference ion was created at the source reference plane $\rho$. at rest, when the ionization process started (time origin).

The ion is detected at $t_{\mathrm{f}}$ from the time origin:

$$
t_{\mathrm{f}}=T+t_{3}+t_{4}
$$

with $t_{4}=D_{4} / v_{3}$ time spent in flight over the last field-free space. A complete first order series development in the small quantities $t_{0}, v_{0} / v_{\mathrm{s}}$ and $s_{0} / D_{1}$ gives:

$$
t_{\mathrm{f}}=t_{\mathrm{f} 0}+\delta t_{1}+\delta t_{2}+\delta t_{3}
$$

where $\delta t_{1}, \delta t_{2}$, and $\delta t_{3}$ are flight time deviations for an ion with initial parameters $s_{0}, t_{0}$, and $v_{0}$, respectively, related to the reference ion:

$$
\begin{aligned}
t_{\mathrm{f} 0}= & T+(2 m)^{1 / 2}\left[\frac{U_{\mathrm{f}}^{1 / 2}-U_{\mathrm{s}}^{1 / 2}}{z e F_{3}}+\frac{D_{4}}{2 U_{\mathrm{f}}^{1 / 2}}\right] \\
\delta t_{1}= & \left\{\left[\frac{1-z e F_{3} D_{4}}{m v_{f}^{2}}\right]\left[\frac{m v_{s}^{2}}{z e F_{3}}+D_{3}(1+k)-v_{\mathrm{s}} T\right] / v_{\mathrm{f}}\right. \\
& \left.-\frac{m v_{\mathrm{s}}}{z e F_{3}}\right\} \frac{s_{0}}{2 D_{1} \sigma^{2}}
\end{aligned}
$$

$$
\begin{aligned}
& \delta t_{2}=\left(\frac{v_{\mathrm{s}}}{v_{\mathrm{f}}}\right)\left[1-\frac{z e F_{3} D_{4}}{m v_{\mathrm{f}}^{2}}\right] t_{0} \\
& \delta t_{3}=-\frac{m v_{\mathrm{s}} v_{0}}{v_{\mathrm{f}} z e F_{1}}\left[1-\frac{z e F_{3} D_{4}}{m v_{\mathrm{f}}^{2}}\right]
\end{aligned}
$$

The final energy and velocity result from the relation

$$
U_{\mathrm{f}}=m v_{\mathrm{f}}^{2} / 2=U_{\mathrm{s}}+z e F_{3}\left[D_{3}+D_{\mathrm{s}}-T\left(2 U_{\mathrm{s}} / m\right)^{1 / 2}\right]
$$

Simultaneous arrival at the detector of the ions created at different moments and that possess different initial velocities is possible by canceling simultaneously $\delta t_{2}$ and $\delta t_{3}$, which happens when

$$
m v_{\mathrm{f}}^{2}=z e F_{3} D_{4}
$$

This statement, which was established for ions that originate from solid surfaces with initial velocity components directed only toward the detector, is also valid for ions produced from gaseous samples. For ions with initial velocities directed against the electric field sense, the turnaround time must be added simply by changing the sign of $v_{0}$ in (12). If the remaining term in $s_{0}$ is unimportant for ions from surfaces, the violation of "space focusing" may be troublesome for ions produced from gas molecules. To optimize the instrument in such a case the three contributions in $v_{0}, s_{0}$, and $t_{0}$ to the packet final size should be balanced [6].

Only ions inside the section $D_{3}$ at the time the field $F_{3}$ is established will be focused. The ions that just entered into the $D_{3}$ section are focused best when the PSPF field is established, rather than near its detector side end. Therefore, the mass range of the ions that undergo PSPF will be confined to the interval:

$$
\begin{aligned}
m_{\mathrm{sin}} & =2 U_{\mathrm{s}} T^{2} /\left(D_{\mathrm{s}}+D_{3}\right)^{2} \\
m_{\max } & =2 U_{\mathrm{s}} T^{2} / D_{\mathrm{s}}^{2}
\end{aligned}
$$

In the last expression the small correctiion due to the packet length was neglected.

The velocity-time focusing condition (13) is satisfied for a mass close to $m_{\max }$ because these ions typically exhibit the poorest resolution. Then $U_{\mathrm{f}} \approx U_{\mathrm{s}}+z e F_{3} D_{3}$ and the voltage $V_{\text {appl }}$ to be applied across the PSPF region can be estimated to be

$$
V_{\text {appl }}=V_{\mathrm{ac}} /\left(0.5 D_{4} / D_{3}-1\right)
$$

where $V_{\mathrm{ac}}$ is the overall ion source accelerating voltage.

The instrument resolution $R$ may be estimated from first order terms accounted for in the packet length with the formula

$$
R=t_{\mathrm{f} 0} /\left[2\left(\left|\delta t_{1}\right|+\left|\delta t_{2}\right|+n\left|\delta t_{3}\right|+\delta t_{\mathrm{e}}\right)\right]
$$




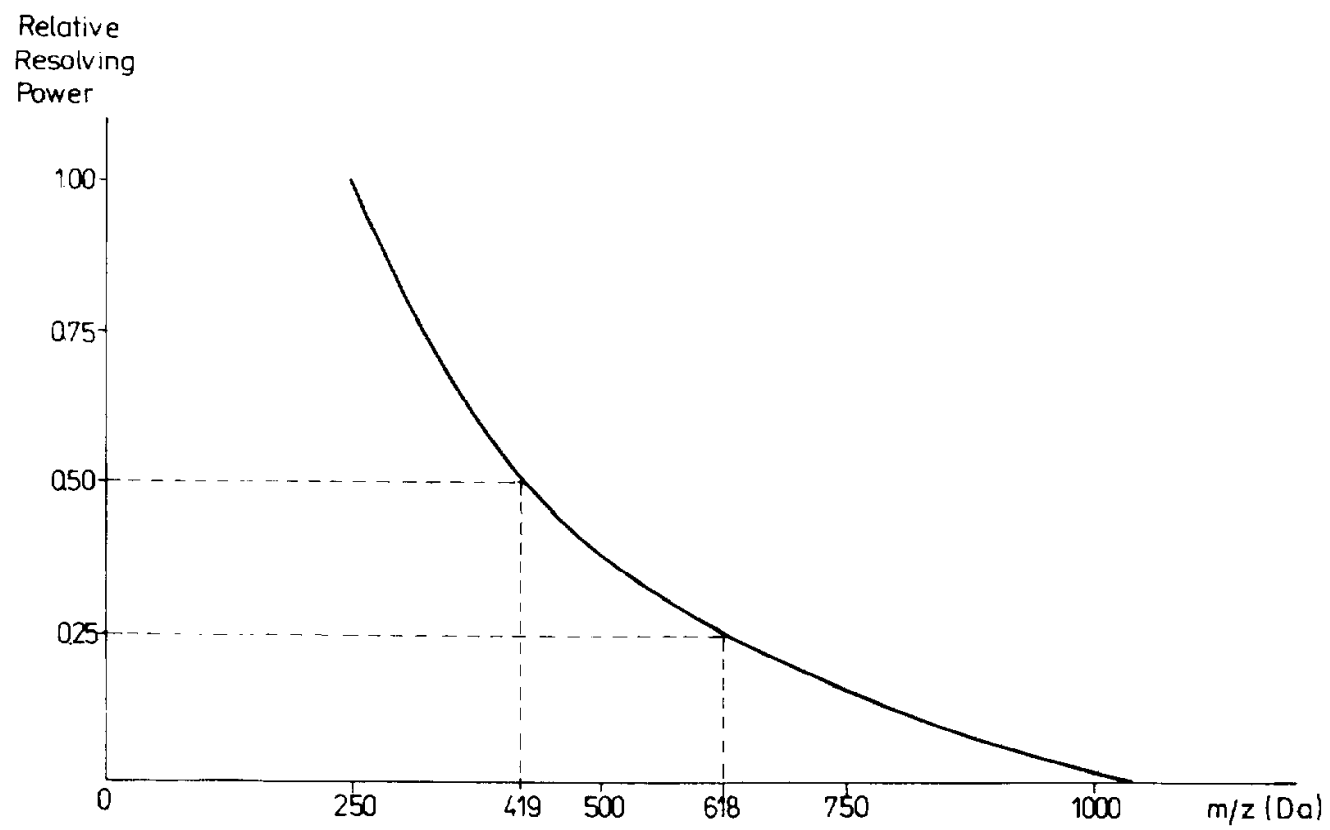

Figure 2. Resolving power over the mass range where PSPF acts. The resolving power for the trontier mass $\left(240.85 u\right.$ ) was taken as unity; the components related to $s_{0}$ and $v_{0}$ are neglected.

Here $\delta t_{\mathrm{c}}$ is the detector time resolution. If the initial ion distributions are rectangular of widths $s_{0}$ in space, $t_{0}$ in time, and $v_{0}$ in velocities, eq 17 gives the TOFMS resolution at the base of the peak. For ions that come from a surface $n=1$, whereas for gas samples $n$ should be substituted by 2 to account for turnaround time. For realistic initial velocity distributions, $v_{0}$ is to be taken as the velocity of the fastest ions with nonnegligible population.

The flight time of the reference ion $t_{f 0}$ as a function of the mass represents the law for mass scale calibration. It is expressed through constants to be determined from known flight times of at least five ion masses:

$$
\begin{aligned}
t_{i 0}= & a_{0}+a_{1} m^{1 / 2}\left[\left(1-a_{2} / m^{1 / 2}\right)^{1 / 2}\right. \\
& \left.-a_{3}+a_{4} /\left(1-a_{2} / m^{1 / 2}\right)^{1 / 2}\right]
\end{aligned}
$$

For the specific case of the instrument of ref 5 , if ions of $m_{\max } / z=1050 \mathrm{u}$ with $U_{3}=7 \mathrm{keV}$ are admitted in the PSPF region, for $\sigma^{2}=14$, the foregoing formulas give $V_{\text {appl }}=732.98 \mathrm{~V}, T=2.3064 \mu \mathrm{s}, m_{\mathrm{min}} / z=$ $241 \mathrm{u}$ (more precisely $240.85 \mathrm{u}$ ). If the focusing condition eq 13 is satisfied for ions of $m / z=1048 \mathrm{u}$, for $V_{\text {appl }}=732.92 \mathrm{~V}$ and $\delta t_{*}$ is neglected, the obtained resolution of 2900 would be limited to this value only due to the first order term in $s_{11}$. Thus the total thick- ness of the ionization region-surface and field nonuniformities included-results from eq 17: $s_{0}=5.5$ $\times 10^{-2} \mathrm{~mm}$.

The capability of the PSPF method is illustrated by the mass dependence of the resolving power when the $t_{0}$ term dominates. The curve of Figure 2 was traced by taking the resolving power for the smallest stillfocused mass $m_{\min }$ as unity. The resolution is increased by a factor of 2 at $m / z=419 u$ and by a factor of 4 at $m / z=618 \mathrm{u}$.

The derived formulas are useful for implementation of PSPF. They allow calculation of the applied voltage, the time delay to focus a selected mass (simpler than those of ref 4), the mass scale, and the theoretical resolution over the focused mass range for a given set of ion formation conditions.

\section{References}

1. Muga, M. L. Anal. Instrum. 1987, 16, 31.

2. Kinsel, G. R.; Johnston, M. V. Int. I. Mass Spectrom. Ion Processes 1989, 91, 157.

3. Yefchak, G. E.; Enke, C. C.; Holland, J. F. Int. J. Mass Spectrom. lon Processes 1989, 87, 313.

4. Kinsel, G. R.; Mowry, C. D.; McKeown, P. J.; Johnston, M. V. Int. J. Mass Spectrom. Ion Processes 1991, 104, 35.

5. Kinsel, G. R.; Grundwuermer, J. M.; Grotemeyer, J. J. Am. Soc. Mass Spectrom. 1993, 4, 2.

6. Ioanoviciu, D. In Advances in Electronics and Electron Physics, Vol. 73; Hawkes, P. W., Ed.; Academic Press: New York, 1989: pp $1-82$. 